This article was downloaded by: [Universiteit Antwerpen]

On: 20 November 2013, At: 00:21

Publisher: Taylor \& Francis

Informa Ltd Registered in England and Wales Registered Number: 1072954 Registered office: Mortimer House, 37-41 Mortimer Street, London W1T 3J H, UK

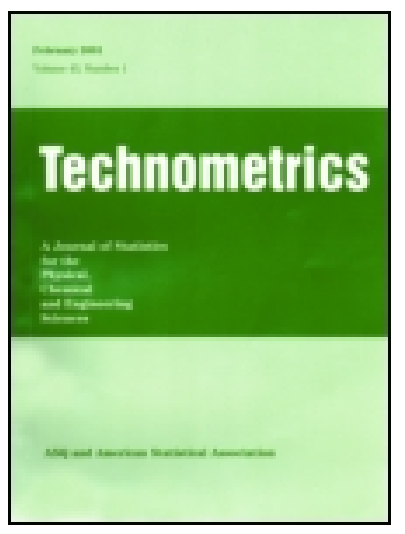

\title{
Technometrics
}

Publication details, including instructions for authors and subscription information: http:// www. tandfonline.com/loi/ utch20

\section{Comment: Enhancing the Search for Compromise Designs}

\author{
Bradley J ones ${ }^{\text {a }}$ \\ a J MP Division, SAS, Cary, NC and Universiteit Antwerpen, Antwerpen, Belgium \\ Published online: 26 Aug 2013.
}

To cite this article: Bradley J ones (2013) Comment: Enhancing the Search for Compromise Designs, Technometrics, 55:3, 278-280, DOI: 10.1080/00401706.2013.804444

To link to this article: http:// dx.doi.org/ 10.1080/00401706.2013.804444

\section{PLEASE SCROLL DOWN FOR ARTICLE}

Taylor \& Francis makes every effort to ensure the accuracy of all the information (the "Content") contained in the publications on our platform. However, Taylor \& Francis, our agents, and our licensors make no representations or warranties whatsoever as to the accuracy, completeness, or suitability for any purpose of the Content. Any opinions and views expressed in this publication are the opinions and views of the authors, and are not the views of or endorsed by Taylor \& Francis. The accuracy of the Content should not be relied upon and should be independently verified with primary sources of information. Taylor and Francis shall not be liable for any losses, actions, claims, proceedings, demands, costs, expenses, damages, and other liabilities whatsoever or howsoever caused arising directly or indirectly in connection with, in relation to or arising out of the use of the Content.

This article may be used for research, teaching, and private study purposes. Any substantial or systematic reproduction, redistribution, reselling, loan, sub-licensing, systematic supply, or distribution in any form to anyone is expressly forbidden. Terms \& Conditions of access and use can be found at http:// www.tandfonline.com/page/terms-and-conditions 
represents the best of current industry practice. Carry out a "natural experiment" by observing the degree to which professionals adopt the advice and evaluate potential correlations with measures of their effectiveness in using experimentation to improve an engineering system.

4. Develop a set of experimental scenarios that invite and enable repeated cycles of problem reframing and experimentation. Have subjects work to accomplish the objectives of system improvement and system modeling while "talking aloud" about the similarities they are able to discover in an effort to reduce the dimensionality and promote scalability of their results. Based on what such experiments reveal, bring statisticians and cognitive psychologists together to devise methods that make learning from data more effective despite the difficulties typically present in authentic experimentation contexts.

Many of these proposed research projects would require a mix of statistical and social science methods. I think this would be a natural and welcome development. As Box (1999) noted, "In the context of iterative learning, optimizations of separate designs will necessarily be sub-optimizations. It is the investigation itself, involving many designs and analyses, that must be regarded as the unit, and the success of the investigation must be regarded as the objective" (p. 21). To realize this vision, researchers will have to explore the influence of our statistical tools, methods, and theories on the fundamentally human and social process of learning through experimentation.
I submit that, under the right conditions, the article "Experimental Design for Engineering Dimensional Analysis" could be a key, early step in novel program of research.

\section{ACKNOWLEDGMENTS}

The author benefited from communications with Edward M. Greitzer, Kim J. Vandiver, and Christopher L. Magee regarding scale modeling in aeronautical engineering, automotive engineering, and naval architecture, respectively. Support of the SUTD-MIT International Design Centre was essential to the author.

\section{ADDITIONAL REFERENCES}

Box, G. E. P. (1999), "Statistics as a Catalyst to Learning by Scientific Method: Part 2-A Discussion," Journal of Quality Technology, 31, 1629. [277,278]

Bridgman, P. W. (1927), The Logic of Modern Physics, New York: Beaufort Books. [275]

Davis, T. P. (2013), "Comment: Dimensional Analysis in Statistical Engineering," Technometrics, 55, 271-275 [277]

Lewis, E. V. (1988), Principles of Naval Architecture (Second Revision), Volume II-Resistance, Propulsion and Vibration, Jersey City, NJ: Society of Naval Architects and Marine Engineers (SNAME). [276]

Luce, D. R. (1971), "Similar Systems and Dimensionally Invariant Laws," Philosophy of Science, 38, 157-169. [275]

Savoie, T. B., and Frey, D. D. (2012), "Detecting Mistakes in Engineering Models: The Effects of Experimental Design," Research in Engineering Design, 23, 155-175. [277]

Sonin, A. A. (2003), "A Generalization of the Pi Theorem and Dimensional Analysis," PNAS, 101, 8525-8526. [275]

Tropea, C., Yarin, A. L., and Foss, J. F. (eds.) (2007), Springer Handbook of Experimental Fluid Mechanics (Vol. 1), Berlin: Springer-Verlag. [276]

\title{
Comment: Enhancing the Search for Compromise Designs
}

\author{
Bradley JONES \\ JMP Division, SAS, Cary, NC \\ and \\ Universiteit Antwerpen \\ Antwerpen, Belgium \\ (Bradley.Jones@jmp.com)
}

First I want to congratulate the authors for producing this important article. It directly addresses problems that engineers and scientists often experience in collaborating with statisticians. That is, statisticians' general preference for empirical models. In the physical sciences and many engineering fields, there is substantial theory that supports models with terms having direct meaning relating to cause and effect.

Much of the literature in design of experiments either implicitly or explicitly assumes that the model to be fit will be a lower-order polynomial approximation of the underlying sys- tem or process behavior. Such models are often quite useful over the range of experimentation but they do not allow for extrapolation and they may not provide much insight into the actual mechanism controlling the system or process.

C) 2013 American Statistical Association and the American Society for Quality

TECHNOMETRICS, AUGUST 2013, VOL. 55, NO. 3 DOI: $10.1080 / 00401706.2013 .804444$ 
Understanding this mechanism may be as important to an engineer or scientist as precise predictions, so an empirical modeling approach is often unsatisfying. The unfortunate outcome of a statistician's stubborn insistence on the use of designs developed for fitting simple empirical models may be the rejection of DOE in its entirety.

Of course, models that make use of dimensional analysis (DA) still have an empirical flavor. Still, the fact that both sides of the model equation are either dimensionless or have the same units gives these models face validity. Providing a recipe that a statistician can use in collaboration to create such a model is a clear contribution of this article.

The authors note that design for DA involves factors that are all quantitative. Certainly, the method requires a quantitative response and quantitative factors with the appropriate units. It seems to me that such studies can also consider sources of noise, which are often categorical such as machines or suppliers. Similarly, blocking may also be useful to separate the variability due to day-to-day or lot-to-lot variation.

My favorite feature of this article is the multiobjective design approach that hedges one's bets on the DA approach. As the authors point out, it is certainly possible to leave out a critical variable in any particular application of DA. If this happens, the design and analysis based solely on the DA may have limited utility. At the possible cost of a few extra runs, the authors provide a compromise design that allows for efficient fits of both an empirical model in the raw factors and the DA model.

The authors consider a continuum of designs going from one that is optimal for the DA model to one that is optimal for fitting a specified empirical model in the raw factors. The design that is optimal for the DA model has an efficiency of $100 \%$ for that model and a much lower efficiency for the empirical model. Conversely, the design that is optimal for the empirical model is $100 \%$ efficient for that model but far less efficient for the DA model. Creating an objective function that is a weighted average of the two efficiencies allows for the discovery of acceptable compromise designs. While such designs are not optimal by either criterion, they may perform well with respect to both criteria.

The authors find a design for a specific weighted average of efficiencies using the coordinate exchange algorithm. This algorithm uses a greedy hill climbing approach. So, while individual designs found using coordinate exchange are usually quite efficient, there is no guarantee that the algorithm will not get stuck in some local optimum. So, most applications of coordinate exchange employ multiple random starts in an effort to find or at least get closer to the global optimum. The authors advocate creating a "w-trace" showing the efficiency by each criterion for 31 pairs of weights where each weight pair sums to 1 . For example, the weight for the DA efficiency might be 0.9 while the weight for the empirical design would then be 0.1 . For each of these 31 pairs of weights, they run coordinate exchange for 100 random starts and choose the design having the best efficiency compromise for each of the 31 pairs. The w-trace then plots the efficiency value for each criterion for every weight pair. Ideally, the w-trace is monotone increasing for one criterion and monotone decreasing for the other. However, because 100 random starts are not sufficient to find the best compromise design for each weight pair, the w-traces in the article are jagged. The au- thors point out that this problem could be handled by employing more random starts but they choose to bound the computational work. For each problem, they compute 3100 designs in all and plot the efficiencies of the most efficient design for each of the 31 weight pairs.

My objective in this discussion is to contribute to the authors' methodology by suggesting an alternative approach for creating the w-trace with the same computational effort. My approach is inspired by the method proposed by Sambo, Borrotti, and Mylona (in press). Briefly, I consider 3100 weight pairs for a specified design problem. For the first two starts, the algorithm generates a design for the weight pairs $[0,1]$ and $[1,0]$, respectively. The individual criterion values for these two end-point designs are then used to construct the efficiency measures for subsequent starts. For each such start, a random weight, w, is chosen from the interval $[0,1]$. The weight pair is $[w, 1-w]$. Rather than a random starting design, the algorithm uses a randomly chosen design from the set of previously generated designs. The solution to a previous design problem is likely to be much better than the objective function value from a random starting design. But, because the weight pair changes from one start to the next, a previously generated design is unlikely to be optimal for the weight pair of new scenario. This makes use of the variable neighborhood search idea that is current in the optimization community.

The algorithm requires keeping a list of the previously generated designs as well as their efficiencies by each criterion and the weights. The next task is to find the set of nondominated designs. A design is in the set of nondominated designs if there is no other design with higher efficiencies for both criteria. These designs comprise a Pareto frontier. Lu, Anderson-Cook, and Robinson (2011) introduced the idea of using Pareto frontiers in the context of optimal designs for multiple objectives. A consequence of producing the Pareto frontier is that the w-trace of

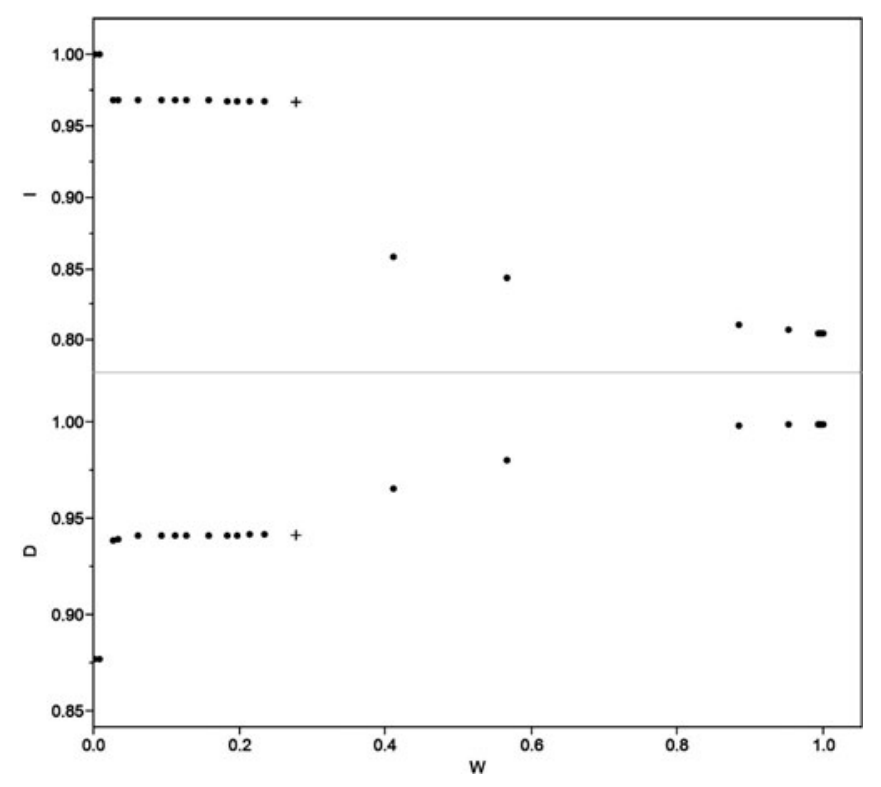

Figure 1. Pareto frontier of designs representing a trade-off of Doptimality versus I-optimality. The point designated with a plus sign represents the compromise design. 
Table 1. D-optimal, compromise, and I-optimal designs for the 12-run, three-factor example

\begin{tabular}{|c|c|c|c|c|c|c|c|c|c|}
\hline Run \# & $\mathrm{X} 1$ & $\mathrm{X} 2$ & X3 & $\mathrm{X} 1$ & $\mathrm{X} 2$ & X3 & $\mathrm{X} 1$ & $\mathrm{X} 2$ & X3 \\
\hline 2 & 1 & 1 & -1 & -1 & 1 & -1 & -1 & -1 & -1 \\
\hline 3 & 1 & -1 & 1 & -1 & -1 & -1 & 1 & -1 & 1 \\
\hline 4 & 1 & -1 & -1 & 1 & -1 & -1 & 1 & 1 & -1 \\
\hline 7 & -1 & -1 & 1 & -1 & 0.266 & 1 & 0.1684 & 1 & 1 \\
\hline 8 & -1 & -1 & -1 & -1 & -1 & 0.152 & 1 & 0.31209 & 0.31209 \\
\hline 9 & -0.00235 & -1 & 0.00235 & -0.176 & 1 & 0.213 & -0.31209 & 0.31209 & -1 \\
\hline 10 & -0.00235 & 1 & 0.00235 & 1 & -0.176 & 0.213 & -0.31209 & -1 & 0.31209 \\
\hline 11 & 1 & 0 & 0.00186 & -0.037 & -0.037 & -1 & 0.09255 & -0.09255 & -0.09255 \\
\hline
\end{tabular}

Note that the D-optimal design has all eight of the cube vertices while the I-optimal design has only four. The compromise design has five vertex points.

the designs on the frontier will be monotone decreasing for one criterion and monotone increasing for the other.

As a demonstration of the efficacy of this approach, consider the problem of finding a good compromise between Doptimality and I-optimality for a three factor, 12 run, response surface design (i.e., one for the estimation of a full quadratic model). Let the design region for this problem be the cube $[-1,1]^{3}$. There are 10 parameters in the model, so finding the best 12-run design by each criterion requires the consideration of all values on the interval $[-1,1]$. Therefore, I also employ the coordinate exchange algorithm, but I use a continuous optimizer for the choice of each coordinate rather than discretizing the interval or limiting my choices to the values $-1,0$, and 1 .

The w-trace of Pareto frontier of designs is shown in Figure 1. The I-optimal design, associated with a weight, $w$, of zero, is more than $87 \%$ D-efficient. By comparison, the D-optimal design, with $w=1$ is slightly more than $80 \%$ I-efficient. The compromise design designated with a plus sign has $w=0.276$, which is slightly less than 95\% D-efficient and nearly $97 \%$ I-efficient. These three designs appear in Table 1.

Both traces are monotone as desired though there are substantial gaps in the w-trace where no nondominated design is found.

\section{SUMMARY}

Scale invariance is an important feature in statistics as well as science and engineering. The authors have provided a valuable service to the discipline of DOE by showing how to apply DA in problems where the raw factors are quantitative with units and the measured response also has units. By matching units on each side of a model, or better yet, making both sides without dimension, the resulting model may sometimes have validity outside the ranges of the experimental data.

Applications showing how to incorporate blocking and categorical factors in such settings would be welcome.

Finally, I believe the w-trace idea is useful but the authors' implementation can be improved without additional computational effort.

\section{ADDITIONAL REFERENCES}

Lu, L., Anderson-Cook, C. M., and Robinson, T. J. (2011), "Optimization of Design Experiments Based on Multiple Criteria Utilizing a Pareto Frontier,' Technometrics, 53, 353-365. [279]

Sambo, F., Borrotti, M., and Mylona, K. (in press), "A Coordinate-Exchange Two-Phase Local Search Algorithm for the D- and I-optimal Design of Split-Plot Experiments," Computational Statistics and Data Analysis. [279] 